\title{
Tailoring of dendritic microstructure in solidification processing by crucible vibration
}

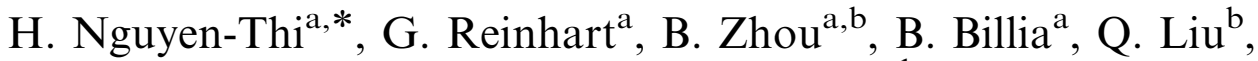 \\ T.P. Lyubimova ${ }^{c}$, B. Roux ${ }^{\mathrm{d}}$ \\ ${ }^{a}$ L2MP, UMR CNRS 6137, Université Paul Cézanne-Aix-Marseille III, Case 142, 13397 Marseille Cedex 20, France \\ ${ }^{\mathrm{b}} N M L C, C A S$, Beijing 100080, China \\ ${ }^{\mathrm{c}}$ Institute of Continuous Media Mechanics UB RAS, Perm, Russia \\ ${ }^{\mathrm{d}}$ Laboratoire de Modélisation en Mécanique, Marseille, France
}

Available online 21 December 2004

\begin{abstract}
We analyzed the effects of both natural convection and forced flows on solid-liquid interface morphology during upward Bridgman solidification of metallic alloys. Experiments were carried out on $\mathrm{Al}-3.5 \mathrm{wt} \% \mathrm{Ni}$ alloy, for a cylindrical sample. The influence of natural convection induced by radial thermal gradient on solidified microstructure was first analyzed as a function of the pulling rate. Then, the influence of axial vibration on solidification microstructure was experimentally investigated by varying vibration parameters (frequency and amplitude). Experimental results demonstrated that vibrations could be used to either attenuate fluid flow in the melt and obtain a uniform dendritic pattern or to promote a fragmented dendritic microstructure. However, no marked effect was observed for cellular growth. This pointed out the critical role of the mushy zone in the interaction between fluid flow and solidification microstructure.
\end{abstract}

(C) 2004 Elsevier B.V. All rights reserved.

PACS: $81.05 . \mathrm{Bx} ;$ 81.30.Fb; 47.27.Bp; 47.27.Hw; 47.27.Te

Keywords: A1. Convection; A1. Dendrites; A1. Directional solidification; A1. Segregation; A1. Stirring; A2. Columnar-equiaxed transition

\footnotetext{
${ }^{*}$ Corresponding author. Tel.: + 33491282893 ; fax: +33491288775 .

E-mail address: henri.nguyen-thi@12mp.fr (H. Nguyen-Thi).
}

\section{Introduction}

During directional solidification of a binary alloy, a sample of initial solute concentration $C_{0}$ is pulled at an imposed velocity $V$ in a constant thermal gradient $G_{L}$. Directional solidification of 
binary alloy presents two major features. First of all, owing to the solubility difference of the two phases, solute is rejected in the liquid during the phase transition when the partition coefficient $k$ is less than unity ( $k$ is defined by the ratio at the interface of the solid composition to the liquid composition). Consequently, solidification creates an exponential solute distribution in the melt and thus local liquid density depends on both thermal and solutal fields. The second main feature is the Mullins-Sekerka instability [1] which drives the breakdown of the planar solid-liquid interface and leads to the formation of cells and dendrites, which are described by suitable shape parameters such as primary and secondary spacings and tip radius. Characteristic relationships have been proposed to relate those parameters to the processing conditions in pure diffusive transport regime $[2,3]$. However, natural buoyancy plays an important role for most real solidification processes and may interact with the microstructure significantly to modify these relationships [4]. Besides, between the fully solid and liquid phases cells and dendrites form a solid-liquid region called "mushy zone", where both phases coexist. For fluid mechanics, the mushy zone can be modeled as an intermediate porous medium through which the melt flows [5].

In Bridgman solidification, one way to prevent thermosolutal convection is to perform the solidification in a both thermal (i.e. vertical upward solidification) and solutal (i.e. rejected solute denser than solvent) stabilizing configuration. Nevertheless, strong convective fluid flow driven by residual radial thermal gradient can be dominant when the growth velocity is slow enough [6]. Indeed, as a mere consequence of gravity, melt made denser by rejected solute moves towards any depressed region of the front, which creates a zone of higher concentration where solidification is further retarded. In real experiments, the initial depression of the solidification front is generally located at the periphery of the sample, due to the difference of thermal conductivity between liquid, solid and crucible. Eventually, the amplification of fluid flow in the vicinity of the solid-liquid interface often leads to large radial solute segregation and hence to a radial gradient of microstructures (cells $\rightarrow$ dendrites $\rightarrow$ eutectic), Fig. 1a. On
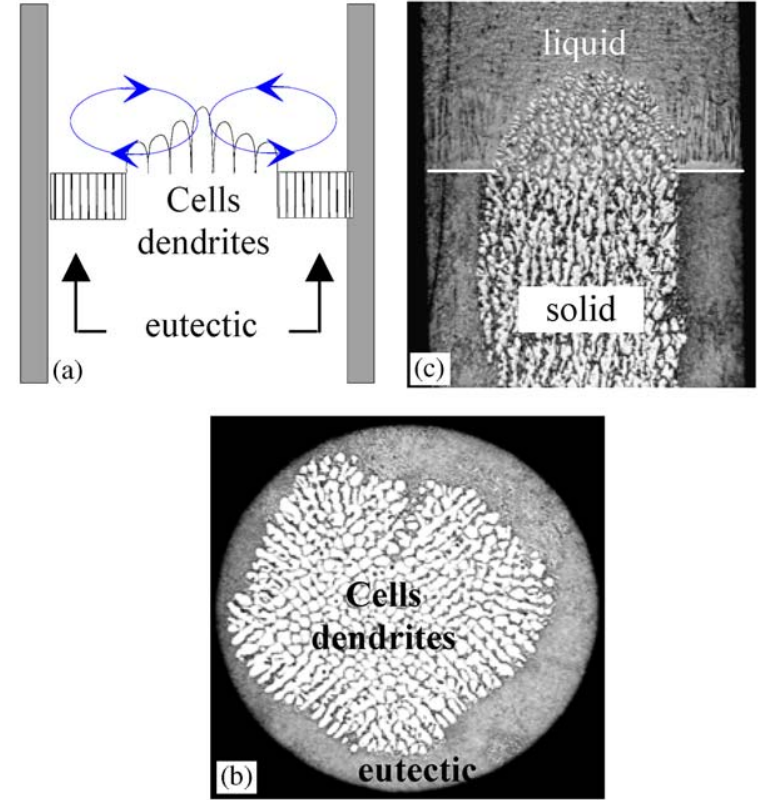

Fig. 1. Distortion induced by convective flow driven by radial thermal gradient: (a) sketch of convective flow and its effects on the solidification front, (b) clustering and (c) steepling phenomena observed on a cross section induced by the fluid flow (Al-3.5\% pds Ni, $G=20 \mathrm{~K} / \mathrm{cm}, V=0.7 \mu \mathrm{m} / \mathrm{s}$ ).

a cross-section (Fig. 1b), the microstructure is not uniform and consists of distinctly separate regions of cellular/dendritic and eutectic growth (localized microstructure due to clustering phenomenon). Moreover, the aluminum cells are 'steepled', i.e. cell tips protrude markedly into the liquid phase with an eutectic front at the base of the solidification front (Fig. 1c).

For bulk sample, the most efficient way to obtain purely diffusive transport in the melt is to carry out solidification in microgravity environment [7,8]. On Earth, the application of an external field during the solidification process, such as magnetic field [9], continuous [10] or alternated [11] rotation around the ampoule axis, can be an effective technique to control fluid flow in the melt. Forced convection is commonly used in many crystal growth processes to improve crystal quality like in Czochralski or floating-zone methods. In the case of Bridgman solidification, there are very few experimental studies $[12,13]$ 
considering the effects of longitudinal vibration on the growth process. As a rule [14], a major effect of vibration is to modify temperature and solute fields in such a way that surfaces of constant density are perpendicular to the vibration axis so that a low radial solute segregation and a rather homogeneous microstructure are expected.

In the present paper, attempts were made to analyze experimentally the effects of vertical vibration on the microstructure and the convective effects. Solidification experiments with and without vertical vibration were performed on Al-3.5 wt $\%$ Ni alloy. The influence of the pulling rate on natural convection was first analyzed. Then, the influence of vertical vibration was investigated by varying the frequency and the amplitude of vibration, for both cellular and dendritic patterns.

\section{Experimental procedure}

Upward directional solidification experiments were carried out on $\mathrm{Al}-3.5 \mathrm{wt} \% \mathrm{Ni}$ alloy with a Bridgman-type furnace, which was previously described in detail [15]. For the present experiments, the inner tube of the furnace was in alumina, whereas previous similar experiments were carried out with a metallic tube. The samples were machined into rods of $8 \mathrm{~mm}$ in diameter and $110 \mathrm{~mm}$ in length and placed in a boron nitride crucible. The whole set was installed at the top of an alumina pulling rod $(20 \mathrm{~mm}$ in outer diameter, $650 \mathrm{~mm}$ in length) and moved by a precise translation device. An electromagnetic vibrator is mounted at the bottom of the alumina rod, which can apply a longitudinal sinusoidal force to the sample. Experiments were carried out for a wide range of frequency $f(10-50 \mathrm{~Hz})$ and amplitude $a$ $(0.25-1.25 \mathrm{~mm})$, in order to obtain various values of vibrational acceleration $g_{\mathrm{vib}}=a(2 \pi f)^{2}$. It is worth noticing that vibrations were applied during the thermal stabilization stage, $15 \mathrm{~min}$ before the beginning of the solidification and during the whole process until the quench.

Experiments were performed in argon ambient at a slight over pressure. After a solidified length of typically $30 \mathrm{~mm}$, the stationary conditions were reached and the residual liquid was quenched by a rapid furnace displacement $(V=2300 \mu \mathrm{m} / \mathrm{s})$. The solidification microstructure was then analyzed by a metallographic procedure in both transverse and longitudinal sections [15].

\section{Experimental results and discussion}

\subsection{Microstructure transitions and effect of natural convection as a function of pulling rate}

Eutectic, cellular and dendritic microstructures were obtained depending on solidification rates within the range $0.1-30 \mu \mathrm{m} / \mathrm{s}$ for a thermal gradient $G=30 \mathrm{~K} / \mathrm{cm}$ (Fig. 2). At very low growth rate $(V=0.1 \mu \mathrm{m} / \mathrm{s})$, a planar eutectic front was obtained and no marked effect of convection was visible, which is expected for these growth conditions (Fig. 2a shows the transverse and longitudinal sections). For higher velocities, within the range $0.3-1.0 \mu \mathrm{m} / \mathrm{s}$, the solidification front was cellular and there was a strong effect of convection, which generated both clustering and steepling phenomena as described in the introduction
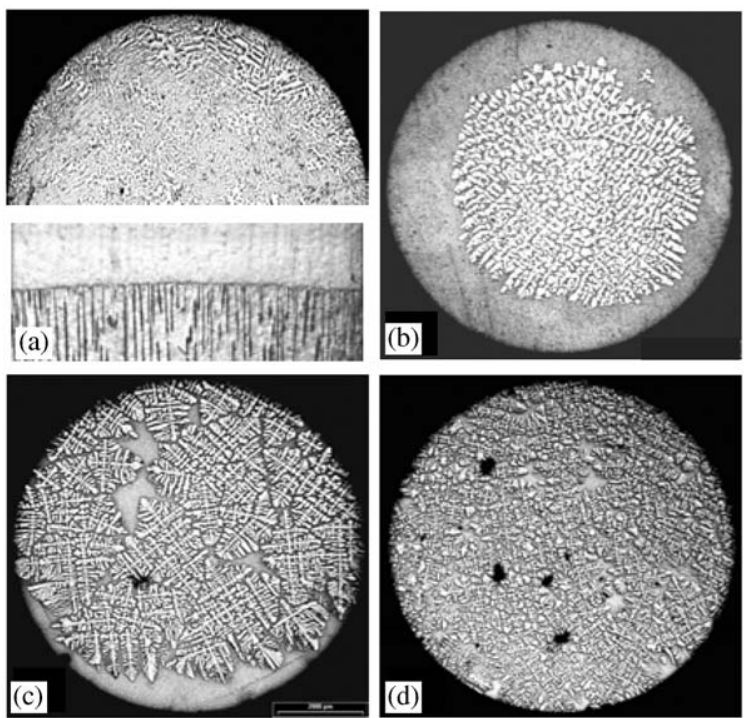

Fig. 2. Pictures showing the solidification microstructure for Al $-3.5 \mathrm{wt} \% \mathrm{Ni}$ alloy, $G=30 \mathrm{~K} / \mathrm{cm}$ and increasing solidification rates, no vibration: (a) $V=0.1 \mu \mathrm{m} / \mathrm{s}$, (b) $V=1 . \mu \mathrm{m} / \mathrm{s}$, (c) $V=7.2 \mu \mathrm{m} / \mathrm{s}$, (d) $V=30 \mu \mathrm{m} / \mathrm{s}$. 
(Fig. 2b). All around dendrite clusters, dendrite arms always grew horizontally in the cross section, towards surrounding eutectic. As the solidification rate was further increased (Fig. 2c), the microstructures became more and more dendritic and the clustering effect of convection progressively decreased (e.g. the eutectic border progressively disappeared). The transition from a strongly disturbed microstructure to an almost homogeneous pattern could be estimated to occur within the range $V=16-24 \mu \mathrm{m} / \mathrm{s}$. For higher pulling rate, an almost homogeneous pattern was observed in cross section (Fig. 2d). It is of value to note that this transition strongly depends on the experimental conditions, in particular the radial thermal symmetry of the furnace that is at the origin of the convection. For example, for the previous experiments described in Ref. [15] performed with a metallic tube, this transition occurred at about $10 \mu \mathrm{m} / \mathrm{s}$. This points out the critical role played by the full temperature profile on this type of convection. Besides, experiments with an other concentration (Al-1.5 wt $\% \mathrm{Ni}$ ) and/ or various thermal gradients $(20$ and $50 \mathrm{~K} / \mathrm{cm})$ gave the same general features [15]. Therefore, this experimental study definitely shows that there is a critical velocity beyond which steepling and clustering phenomena disappear so that solidification produces a uniform dendritic microstructure as predicted by Dupouy et al. [16].

\subsection{Influence of vibrations on cellular and dendritic growth}

Four series of experiments were carried out for Al-3.5 wt $\%$ Ni alloy with the same thermal gradient $(30 \mathrm{~K} / \mathrm{cm})$, for different pulling rates $V=1-3-7.2-9 \mu \mathrm{m} / \mathrm{s}$, to examine the effect of longitudinal sinusoidal vibration on directional solidification. With these values, the solidification microstructure varied from a cellular pattern to a dendritic pattern, which correspond, respectively, to a shallow mushy zone with a very small liquid fraction and a deep mushy zone with a large liquid fraction. For each series of experiments, the influence of vibration level on the solidification microstructure was analyzed as a function of frequency and amplitude of vibrations. It will be shown that the changes induced by vibration on the solidification morphologies strongly depend not only on the level of vibration, but also on the type of microstructure (cellular or dendritic). In the following, we are limiting our discussion to $V=1$ and $7.2 \mu \mathrm{m} / \mathrm{s}$.

For cellular growth $(V=1 \mu \mathrm{m} / \mathrm{s})$, very weak changes were induced by the vertical vibrations as shown in Fig. 3, regardless of the additional vibration acceleration $g_{\text {vib }}$ with respect to natural convection $\left(g_{\text {vib }}=0\right)$. The main effect of vibration was to push the cellular cluster on one side of the transverse section. This feature seemed to be a rule in our experiments, which suggests that vibrations were either not perfectly axial or broke fluid flow symmetry. Further experiments or numerical simulations are required to give a definitive answer to this observation. For the cellular pattern itself, vibrations induced no marked modification, which was probably due to the lack of a porous mushy zone. In that case, the liquid fraction at the interface was very low and fluid flow could not penetrate deep into the mushy zone. Convection could not develop on a large scale in the interior of the mushy zone and remained mainly parallel to the solid-liquid interface [8]. Therefore, the influence of natural and forced convection was expected to be rather limited and the average wavelengths of cellular arrays were within the range $200-300 \mu \mathrm{m}$, regardless of vibration level.

For dendritic growth $(V=7.2 \mu \mathrm{m} / \mathrm{s})$, the changes induced by axial vibrations on the solidification microstructure were significant.
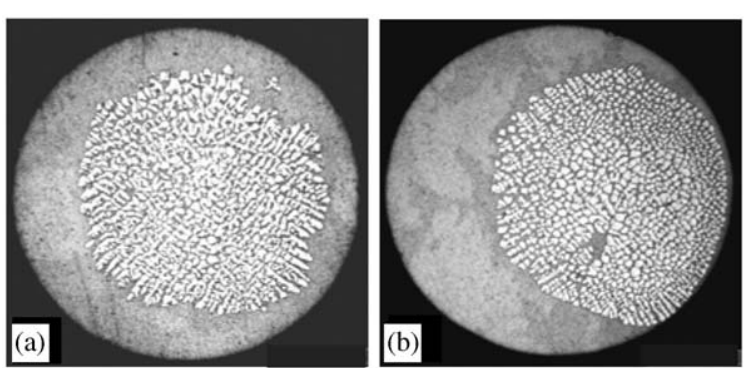

Fig. 3. Cross sections showing the microstructures for Al-3.5 wt $\%$ Ni alloy, $G=30 \mathrm{~K} / \mathrm{cm}, V=1.0 \mu \mathrm{m} / \mathrm{s}$, and various sets of vibration parameters: (a) no vibration, (b) $g_{\text {vib }}=2.5 g_{0}$ $(f=50 \mathrm{~Hz}, a=0.25 \mathrm{~mm})$. 

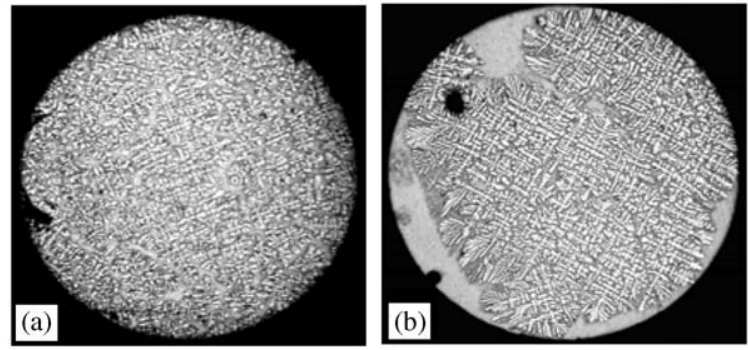

Fig. 4. Cross sections showing the microstructures for Al-3.5 wt $\% \mathrm{Ni}, G=30 \mathrm{~K} / \mathrm{cm}, V=7.2 \mu \mathrm{m} / \mathrm{s}$ and two different sets of vibration parameters given the same value of $g_{\text {vib }}=1.0 g_{0}$ : (a) $f=14 \mathrm{~Hz}, a=1.25 \mathrm{~mm}$, (b) $f=16 \mathrm{~Hz}, a=$ $1.0 \mathrm{~mm}$.

A series of experiments with various level of $g_{\mathrm{vib}}$ from $0.5 g_{0}$ to $2.5 g_{0}$ were carried out. For the lowest value, the microstructure was very similar to the case of natural convection (Fig. 2c). When the vibrational acceleration is about $1.0 g_{0}$, two different cases were obtained, depending on the value of the vibration amplitude. For $(f=14 \mathrm{~Hz}$, $a=1.25 \mathrm{~mm}$ ), the morphology became more homogeneous, with almost no eutectic border (Fig. 4a) while for $(f=16 \mathrm{~Hz}, a=1.0 \mathrm{~mm})$ the microstructure was still strongly disturbed by convection (Fig. 4b). These two experiments were performed with the same experimental procedure and the only difference was the amplitude-frequency parameter set giving the same vibration acceleration. This suggests that the effect of vibrations must be characterized not only by vibrational acceleration $g_{\text {vib }}$ but also by the amplitude $a$. Besides, the microstructure is finer with vibration.

The behavior was quite different for higher levels of vibration. At about $2.3 g_{0}(f=24 \mathrm{~Hz}$, $a=1.0 \mathrm{~mm})$ and $2.5 g_{0}(f=50 \mathrm{~Hz}, a=0.25 \mathrm{~mm})$, the morphologies were fragmented and a great number of clusters of globular cells or dendrites are distributed in a cross section (Fig. 5a and c). Longitudinal sections showed that the growth remained columnar when the vibration level was low whereas the structure was strongly disturbed and a pattern somewhat like equiaxed growth was obtained for higher levels of vibration (Fig. 5b and d). Presently, it is difficult to determine
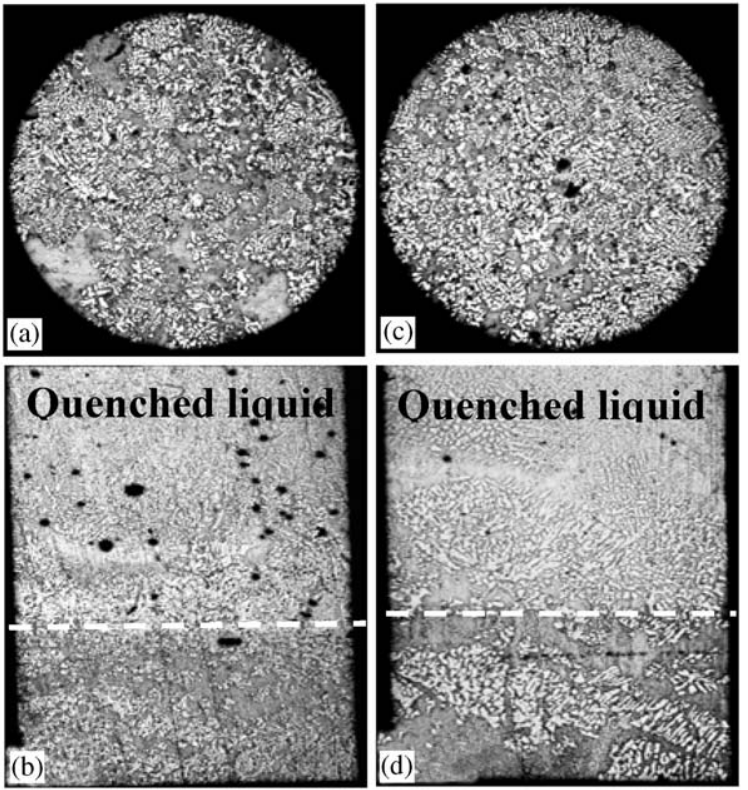

Fig. 5. Cross and longitudinal sections showing the microstructures for Al-3.5 wt $\% \mathrm{Ni}, G=30 \mathrm{~K} / \mathrm{cm} . V=7.2 \mu \mathrm{m} / \mathrm{s}$ and two different sets of vibration parameters: (a, b) $g_{\text {vib }}=2.3 g_{0}$, $f=24 \mathrm{~Hz}, a=1.0 \mathrm{~mm}$, (c, d) $g_{\mathrm{vib}}=2.5 g_{0}, f=50 \mathrm{~Hz}, a=$ $0.25 \mathrm{~mm}$.

whether secondary arms were detached or not from primary stalks during the growth process, which is a key question for the columnar to equiaxed transition during directional solidification.

\section{Conclusion}

Directional solidification experiments were performed under natural and vibration-controlled conditions. The role of processing parameters (pulling rate and level of vibration) was systematically evaluated. The experimental results provide evidence that vibration can strongly modify fluid flow in the melt and thus, act on the solidification morphology. At low growth rates and without vibration, a sharp microstructural transition from cellular/dendritic to eutectic growth was seen from the center to the periphery of a cross section, which is referred to as clustering phenomenon reflecting a large radial 
compositional gradient. This feature disappeared when high enough growth rate was applied and diffusive transport was taking place in the melt.

Under vibration controlled conditions, the response of the solidification front to the vibration depends on the solidification microstructure. The effect was pronounced for dendritic pattern when there was a deep mushy zone: at low level of vibration $\left(g_{\text {vib }}=1.0 g_{0}\right)$, vibration could promote a regular microstructure when the amplitude of vibrations was large enough. The severe radial segregation disappeared, like for samples solidified in microgravity conditions. When the vibration level was above $2 g_{0}$, the microstructure became fragmented and columnar growth was suppressed. In cross section, a large number of clusters of cells or dendrites were distributed. For cellular growth, the effect of vibration was weak and no marked changes were visible on cross or longitudinal sections. To progress further in these experiments, comparison with numerical simulations are now required in order to separate the role of each vibration parameters. At present time, this work is in progress. Preliminary results of numerical simulation already show that vibrational convection is negligible with respect to buoyancy-driven convection as long as a smooth solid-liquid interface is maintained, which confirms the necessity of incorporating the mushy zone in the modeling and suggests that vibrational fluid flow in the porous dendritic mush may be the key factor for the alteration of the solidification microstructure.

\section{Acknowledgements}

The support of the European Space Agency (CETSOL MAP) and the Centre National d'Etudes Spatiales (GDR Phénomènes de Transport et Transitions de Phases en Micropesanteur) is gratefully acknowledged.

\section{References}

[1] W.W. Mullins, R.F. Sekerka, J. Appl. Phys. 35 (1964) 444.

[2] J.S. Langer, Lectures in the theory of pattern formation, in: Chance and Matter, Les Houches Summer School, North Holland, Amsterdam, 1986.

[3] J.D. Hunt, S.Z. Lu, Metall. Mater. Trans. 27A (1996) 611.

[4] S.H. Davis, J. Fluid Mech. 212 (1990) 241.

[5] M.G. Worster, J. Fluid Mech. 237 (1992) 649.

[6] M.H. Burden, D.J. Hebditch, J.D. Hunt, J. Crystal Growth 20 (1973) 121.

[7] B. Drevet, et al., Adv. Space Res. 16 (1995) 173.

[8] B. Drevet, et al., J. Crystal Growth 218 (2000) 419.

[9] D.T.J. Hurle, R.W. Series, Use of a magnetic field in melt growth, in: D.T.J. Hurle (Ed.), Handbook of Crystal Growth, vol. 2a, North Holland, Amsterdam, 1994.

[10] R.N. Grugel, S. Kim, T. Woodward, T.G. Wang, J. Crystal Growth 121 (1992) 599.

[11] J. Wanqi, et al., in: Solidification Processing 97, University of Sheffield, Sheffield, UK, 1997. p. 49.

[12] W. Bingbo, Acta Metall. Mater. 40 (1992) 2739.

[13] R. Caram, M. Banan, W.R. Wilcox, J. Crystal Growth 114 (1991) 249.

[14] G.Z. Gershuni, D.V. Lyubimov, Thermal Vibrational Convection, Wiley, West Sussex, England, 1998.

[15] B.H. Zhou, et al., in: Proceedings of the International Conference on "Advanced Problems in Thermal Convection", Perm, Russia, to be published.

[16] M.D. Dupouy, D. Camel, J. Crystal Growth 183 (1998) 469. 